\title{
Armazenamento de mirtilo 'Bluegem' em atmosfera controlada e refrigerada com absorção e inibição do etileno
}

\author{
Auri Brackmann ${ }^{1}$, Anderson Weber ${ }^{2}$, Ricardo Fabiano Hettwer Giehl ${ }^{3}$, Ana Cristina Eisermann ${ }^{4}$, \\ Cláudia Kaehler Sautter ${ }^{1}$,Emerson Dias Gonçalves ${ }^{5}$, Luis Eduardo Corrêa Antunes ${ }^{6}$
}

\begin{abstract}
RESUMO
O mirtilo é uma fruta rica em polifenóis e antocianinas que apresenta alta atividade antioxidante. No entanto, essa atividade pode ser alterada por condições de armazenamento e processamento dos frutos. O objetivo deste trabalho foi avaliar a manutenção das qualidades físicas e químicas de frutos de mirtilo (Vaccinium ashei Reade) do cultivar Bluegem em diferentes condições de armazenamento. O delineamento experimental utilizado foi o inteiramente casualizado com quatro repetições e unidade experimental composta por $400 \mathrm{~g}$ de frutos. Os tratamentos utilizados foram: [1] armazenamento refrigerado (AR); [2] AR com absorção de etileno por sachês de permanganato de potássio; [3] atmosfera controlada (AC) com 2,0kPa O + 10,0kPa CO ; [4] AC com 2,0kPa O + 15,0kPa CO ; e [5] AC com 10,0kPa O + 15,0kPa CO ; [6] AC com $2,0 \mathrm{kPa} \mathrm{O}_{2}+10,0 \mathrm{kPa} \mathrm{CO}_{2}$ e aplicação de $900 \mu \mathrm{g} \mathrm{kg}^{-1}$ de 1-metilciclopropeno. Os frutos submetidos a todos os tratamentos foram armazenados na temperatura de $0,5^{\circ} \mathrm{C}\left( \pm 0,1^{\circ} \mathrm{C}\right)$. A avaliação da qualidade dos frutos foi realizada após sete semanas de armazenamento mais 84 horas (três dias e meio) de exposição a $20^{\circ} \mathrm{C}\left( \pm 0,2^{\circ} \mathrm{C}\right)$. A melhor conservação de mirtilo 'Bluegem' ocorreu em armazenamento refrigerado, em que se obteve maior acidez titulável, teor de sólidos solúveis totais e porcentagem de frutos firmes, conteúdo de antocianinas e polifenóis totais. Além de menor produção de etileno e $\mathrm{CO}_{2}$. A remoção do etileno da câmara de armazenamento favorece a conservação dos frutos.
\end{abstract}

Palavras-chave: Conservação, etileno, dióxido de carbono, polifenóis, antocianinas.

\section{ABSTRACT}

\section{Storage of 'bluegem' blueberry in controlled atmosphere and cold storage with ethylene scrubbing and inhibition}

The aim of this work was to evaluate storage conditions on the maintenance of the physiochemical qualities in Bluegem blueberry. The experiment was arranged in a complete randomized design , with four replicates and the experimental unit consisted of approximately $400 \mathrm{~g}$ of fruits. Six storage conditions were evaluated as follows: [1] Cold storage (CS); [2] CS with ethylene scrubbing; [3] Controlled atmosphere (CA) with $2.0 \mathrm{kPa} \mathrm{O}_{2}+10.0 \mathrm{kPa} \mathrm{CO}_{2}$; [4] CA with $2.0 \mathrm{kPa} \mathrm{O}_{2}+15.0 \mathrm{kPa} \mathrm{CO}_{2}$; [5] CA with $10.0 \mathrm{kPa} \mathrm{O}_{2}+15.0 \mathrm{kPa} \mathrm{CO}_{2}$; [6] CA with $2.0 \mathrm{kPa} \mathrm{O}_{2}+10.0 \mathrm{kPa} \mathrm{CO}_{2}$ with application of $900 \mu \mathrm{g} \mathrm{kg}{ }^{-1}$ of 1 -methilciclopropene. All treatments were stored at $0.5^{\circ} \mathrm{C}\left( \pm 0.1^{\circ} \mathrm{C}\right)$. Fruit quality was evaluated after seven weeks of storage plus three more days at $20^{\circ} \mathrm{C}\left( \pm 0.2^{\circ} \mathrm{C}\right)$. The best 'Bluegem' blueberry conservation was achieved in cold storage, which gave the highest titratable acidity, total soluble solids and percentage of firm fruit, lowest ethylene and $\mathrm{CO}_{2}$ production as well as the highest anthocyanin and total polyphenolic contents. However, the removal of ethylene in the storage room can prolong fruit conservation.

Key words: Conservation, ethylene, carbon dioxide, polyphenolics, anthocyanins.

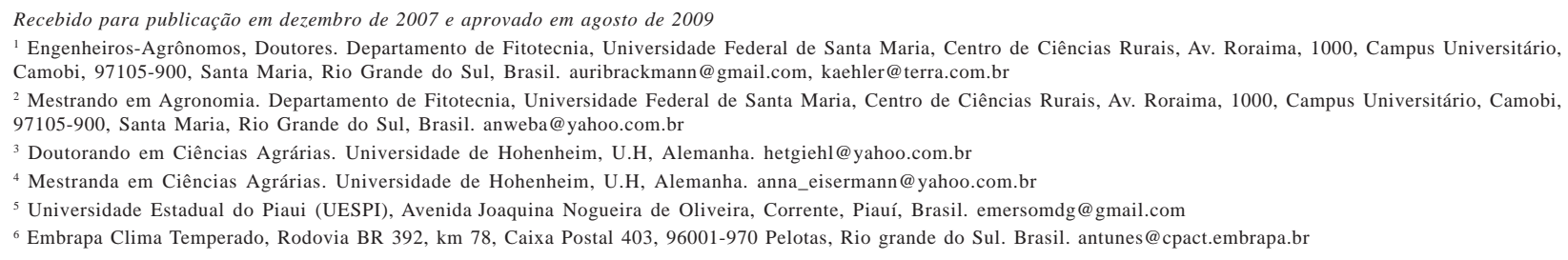




\section{INTRODUÇÃO}

O mirtilo (Vaccinium ashei Reade), pertencente à família Ericaceae, é uma frutífera nativa do Hemisfério Norte, onde é cultivada comercialmente em larga escala, principalmente nos EUA e em alguns países da Europa. No Hemisfério Sul é cultivado comercialmente no Chile. Apesar de ser uma cultura com pouca expressão comercial no Brasil, o potencial de cultivo do mirtilo no Sul do País é significativo.

Os frutos do cultivar Bluegem têm aproximadamente $1,0 \mathrm{~cm}$ de diâmetro e peso médio de $1,3 \mathrm{~g}$, sendo a casca azul-escura e a polpa com grande número de sementes e de pruína (cera natural que envolve a casca). Os frutos destinam-se ao consumo in natura e ao processamento, como congelamento, desidratação ou fabrico de geléias ou licores. O mirtilo é uma fruta rica em polifenóis, antocianinas e apresenta alta atividade antioxidante (Su \& Silva, 2006). Essa atividade antioxidante pode ser impactada por vários fatores, como variedade, estágio de maturação dos frutos, condição de armazenamento e processamento (Su \& Chien, 2007).

O aumento da área cultivada e o bom preço alcançado na comercialização do mirtilo fazem com que aumente o interesse em produzi-la por produtores brasileiros, os quais podem comercializá-la na entressafra dos países tradicionalmente consumidores e produtores. No entanto, existem alguns problemas que impedem o desenvolvimento da cultura na região Sul do Brasil. Um dos entraves da produção de mirtilos refere-se ao fato de que o período máximo de conservação e as condições mais adequadas para a manutenção das características pós-colheita dos frutos são ainda pouco conhecidos.

Devido aos processos fisiológicos do amadurecimento e à deterioração causada por fungos, como Botrytis cinerea, Alternaria sp. e Colletotrichum gloesosporiodes (Milholland, 1995), o tempo de armazenamento dos frutos de mirtilo é limitado.

Segundo Prange et al. (1995), o mirtilo pode ser armazenado por um período superior a seis semanas quando em concentrações de 1 a $10 \% \mathrm{O}_{2}+10$ a $15 \% \mathrm{CO}_{2}$ na temperatura de até de $5^{\circ} \mathrm{C}$. Segundo Westwood (1982), o mirtilo pode ser armazenado por duas semanas a uma temperatura variando entre 2,0 e 4,0 $0^{\circ} \mathrm{C}$, com umidade relativa de 90 a $95 \%$. Para Hardenburg et al. (1986), o mirtilo suporta até duas semanas em temperaturas entre $-0,5$ a $0^{\circ} \mathrm{C}$ e com umidade entre 90 e 95\%. Já Miller et al. (1988) observaram que o mirtilo pode ser armazenado por um período superior a quatro semanas nessas mesmas condições. Schotsmans et al. (2007) verificaram que o mirtilo ‘Centurion’ pode ser armazenado por até seis semanas sem perda da qualidade físico-química.

Portanto, pela indefinição das condições de armazenamento e do período de armazenagem, este trabalho objetivou avaliar a manutenção das qualidades físicas e químicas de frutos de mirtilo do cultivar Bluegem em diferentes condições de armazenamento.

\section{MATERIAL E MÉTODOS}

O experimento foi conduzido no NPP/UFSM (Núcleo de Pesquisa em Pós-colheita da Universidade Federal de Santa Maria) em parceria com a EMBRAPA/ CPACT de Pelotas. Foram utilizados frutos de mirtilo colhidos em pomar comercial, na região de Pelotas, e transportados para Santa Maria. Após a chegada dos frutos ao NPP, realizou-se a homogeneização, pesagem e acomodação das amostras experimentais em bandejas de isopor, abertas, medindo 14,0 x 14,0 x $4,0 \mathrm{~cm}$. Os frutos apresentaram, no momento da colheita, teores de acidez titulável total (ATT) de 10,5meq $100 \mathrm{~mL}^{-1}$; sólidos solúveis totais (SST) de 11,7º Brix; polifenóis totais de 3861,0mg de ácido gálico $\mathrm{L}^{-1} \mathrm{e}$ antocianinas de $1.256,2 \mathrm{mg}$ de malvidina $\mathrm{L}^{-1}$. O delineamento experimental utilizado foi o inteiramente casualizado, com seis tratamentos e quatro repetições, sendo a unidade experimental composta por aproximadamente $400 \mathrm{~g}$ de frutos. As condições de armazenamento avaliadas foram: [1] armazenamento refrigerado (AR); [2] AR com absorção de etileno por sachês de permanganato de potássio; [3] atmosfera controlada (AC) com 2,0kPa de $\mathrm{O}_{2}+10,0 \mathrm{kPa}$ de $\mathrm{CO}_{2}$; [4] AC com 2,0kPa de $\mathrm{O}_{2}+15,0 \mathrm{kPa}$ de $\mathrm{CO}_{2}$; e [5] $\mathrm{AC}$ com $10,0 \mathrm{kPa}$ de $\mathrm{O}_{2}+15,0 \mathrm{kPa}$ de $\mathrm{CO}_{2}$; [6] AC com 2,0kPa de $\mathrm{O}_{2}+10,0 \mathrm{kPa}$ de $\mathrm{CO}_{2}$ e aplicação de $900 \mu \mathrm{g} \mathrm{kg} \mathrm{kg}^{-1}$ de 1 MCP. Os frutos de todos os tratamentos foram armazenados na temperatura de $0,5^{\circ} \mathrm{C}\left( \pm 0,1^{\circ} \mathrm{C}\right)$.

No tratamento com 1-MCP utilizou-se o produto Smartfresh ${ }^{\circledR}$, que contém $0,14 \%$ do princípio ativo, diluído em água destilada e deionizada, ajustando-se a concentração para $900 \mu \mathrm{g} \mathrm{kg}^{-1}$. No tratamento com absorção de etileno $\left(\mathrm{C}_{2} \mathrm{H}_{4}\right)$ utilizaram-se sachês do Always fresh ${ }^{\circledR}$ que contêm "pellets" impregnados com permanganato de potássio. Esses foram colocados no interior das minicâmaras, para absorver o etileno produzido pelos frutos.

Para o armazenamento das amostras, foram utilizadas minicâmaras experimentais de 50 litros, fechadas de forma hermética, nas quais se instalou a condição de atmosfera referente a cada tratamento. Os métodos de estabelecimento das condições de AC e seu controle estão descritos em Brackmann et al. (2008). A temperatura da câmara frigorífica foi regulada automaticamente por meio de termostato eletrônico e também determinada diariamente por meio de termômetros com bulbo de mercúrio de alta resolução.

Após sete semanas de armazenamento, os frutos foram retirados das minicâmaras experimentais e colocados a $20^{\circ} \mathrm{C}\left( \pm 0,2^{\circ} \mathrm{C}\right)$, permanecendo nesta tempera- 
tura por três dias e meio, sendo em seguida analisados em laboratório. As variáveis produção de etileno, respiração, acidez titulável e sólidos solúveis totais foram avaliados conforme descrito em Brackmann et al. (2008). As demais características analisadas neste experimento foram: a) frutos murchos: foram contados os frutos que apresentavam, visualmente, sintomas de murchamento, sendo os dados expressos em porcentagem. b) firmeza dos frutos: foram considerados frutos firmes aqueles que, quando pressionados com a ponta dos dedos, não apresentavam deformação, sendo os dados expressos em porcentagem de frutos firmes. c) perda de massa: determinada pela diferença de peso entre os frutos antes do armazenamento e após a saída dos frutos da câmara, sendo os valores expressos em porcentagem. d) polifenóis totais: a concentração de polifenóis totais foi determinada pelo método colorimétrico descrito por Singleton \& Rossi (1965). Em balão volumétrico de 20mL, adicionaram-se $2 \mathrm{~mL}$ de amostra diluída (1:10), 10mL de reagente de Folin-Ciolcateau diluído e $8 \mathrm{~mL}$ de $\mathrm{Na}_{2} \mathrm{CO}_{3}$ 7,5\%. Completou-se o volume com água destilada e, após duas horas, leu-se a absorbância a 765nm em espectrofotômetro 600 da marca Femto ${ }^{\circledR}$. Para a quantificação foi empregada uma curva padrão com solução de ácido gálico nas seguintes concentrações: 50, 100, 150, 250 e $500 \mathrm{mg} \mathrm{L}^{-1}$. O teor de polifenóis totais foi expresso em equivalentes de ácido gálico $\left(\mathrm{mg} \mathrm{L}^{-1}\right)$ e e ) antocianinas totais: foram determinadas conforme descrito por Di Stefano et al. (1989). A extração das antocianinas foi feita numa solução de eta-nol:água: $\mathrm{HCl}$ (70:30:1), e o conteúdo estimado como malvidina a 531nm em espectrofotômetro 600 da marca Femto ${ }^{\circledR}$, usando um coeficiente de extinção molar de $18,8 \mathrm{~L} \mathrm{~cm}^{-1} \mathrm{~mol}^{-1}$.

Para cada característica avaliada foi efetuada uma análise da variância, sendo as médias comparadas pelo teste de Tukey, com 5\% de probabilidade de erro. As variáveis firmeza, frutos murchos e perda de massa, com distribuição binomial e expressas em porcentagem, foram transformadas pela fórmula arc.sen $\sqrt{\mathrm{x} / 100}$, antes da análise da variância, objetivando obter erros com distribuição normal.

\section{RESULTADOS E DISCUSSÃO}

Após 36 horas de permanência a $20^{\circ} \mathrm{C}$ observou-se um pico de produção de etileno que atingiu 3,06 $\mu \mathrm{L} \mathrm{C}_{2} \mathrm{H}_{4}$ $\mathrm{kg}^{-1} \mathrm{~h}^{-1}$ nos frutos armazenados a 10,0kPa de $\mathrm{O}_{2}+15,0 \mathrm{kPa}$ de $\mathrm{CO}_{2}$. Somente no tratamento em que se aplicou 1MCP não ocorreu o pico de produção de etileno após 36 horas a $20^{\circ} \mathrm{C}$ (Figura $\left.1 \mathrm{~A}\right)$. Warren et al. (1973) reportaram que o etileno participa ativamente na maturação do mirtilo e que a aplicação de ácido 2-cloroetilfosfônico, que é uma formulação sintética de etileno, resultou num avanço da maturação e no período de colheita. A maior produção de etileno, após $12 \mathrm{~h}$ de exposição a $20^{\circ} \mathrm{C}$, foi observada para os frutos submetidos ao armazenamento em atmosfera refrigerada, porém nas avaliações posteriores ocorreu menor produção desse fito-hormônio por tais frutos. Após 84 horas de exposição a $20^{\circ} \mathrm{C}$ os frutos armazenados com $15,0 \mathrm{kPa}$ de $\mathrm{CO}_{2}$ e os que receberam aplicação de 1-MCP apresentaram maior produção de etileno, sendo ainda observada a menor produção de etileno nas condições de AR, o que justifica a melhor manutenção das características físicas e químicas nos frutos armazenados em AR. A aplicação de 1-MCP resultou em aumento progressivo da produção de etileno até a última avaliação, com maior produção após 84 horas de manutenção a $20^{\circ} \mathrm{C}$ em relação às demais condições de armazenamento (Figura 1A).

A produção de $\mathrm{CO}_{2}$ foi maior nos frutos armazenados na condição de $15 \mathrm{kPa}$ de $\mathrm{CO}_{2}$ na avaliação após 12 h a $20^{\circ} \mathrm{C}$ (Figura 1B). Este resultado provavelmente ocorreu em função da liberação do $\mathrm{CO}_{2}$ acumulado nos espaços
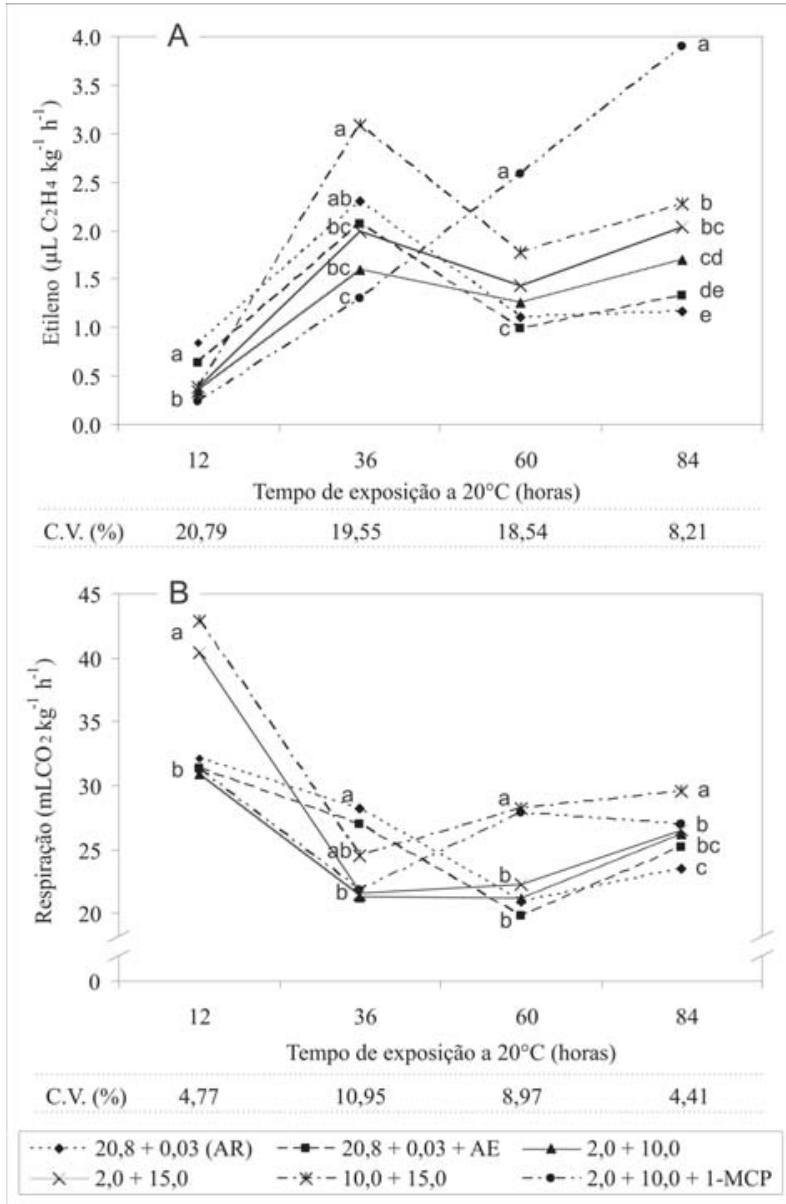

Figura 1. Evolução da produção de etileno (A) e respiração (B) de mirtilo, cultivar Bleugem, mantido por 84 horas a $20^{\circ} \mathrm{C}$, após sete semanas em diferentes condições de armazenamento a $0,5^{\circ} \mathrm{C}$. Médias seguidas de mesma letra na vertical não diferem entre si pelo teste de Tukey $(\mathrm{p}<0,05)$. 
intercelulares da polpa devido ao longo período de exposição dos frutos a alta concentração de $\mathrm{CO}_{2}$. Este acúmulo pode ter superestimando a produção de $\mathrm{CO}_{2}$ na primeira avaliação após a armazenagem. Entretanto, a alta pressão parcial de $\mathrm{CO}_{2}$ não conseguiu inibir suficientemente a produção de etileno. Alta produção de etileno e, consequentemente, aumento da produção de $\mathrm{CO}_{2}$ provocam aumento no metabolismo dos frutos durante sua exposição a $20^{\circ} \mathrm{C}$, acarretando grandes alterações das características físicas e químicas (Watkins, 2000) e, com isso, diminuindo o período de vida útil do produto.

Os maiores teores de acidez foram observados nos frutos que receberam aplicação de 1-metilciclopropeno e nos frutos armazenados em atmosfera refrigerada (Tabela 1). Harb \& Streif (2006) também verificaram que a melhor manutenção da acidez no cultivar Bluecrop se deu em condições de atmosfera refrigerada e em condições de atmosfera controlada com no máximo $12 \mathrm{kPa}$ de $\mathrm{CO}_{2}$. Nas condições de armazenamento em que a concentração de $\mathrm{CO}_{2}$ foi alta, observou-se aumento na taxa respiratória, ocorrendo assim maior metabolismo. Conseqüentemente, isso resultou em maior utilização dos ácidos orgânicos presentes nos frutos, diminuindo a acidez titulável total. Harb \& Streif (2006) verificaram o mesmo efeito quando os frutos foram armazenados em concentrações acima de $12 \mathrm{kPa}$ de $\mathrm{CO}_{2}$. A acidez titulável nos frutos de mirtilo cultivar Duke também se reduziu quando o $\mathrm{CO}_{2}$ foi elevado de 12 para $24 \mathrm{kPa}$ (Harb \& Streif, 2004). A maior acidez nos frutos mantidos em AC com aplicação 1-MCP é resultado do bloqueio da síntese de etileno (Sisler \& Serek, 1999), resultando em diminuição do processo respiratório e assim diminuindo a degradação dos ácidos e mantendo a qualidade inicial dos frutos (Wills \& Ku, 2002).

Os sólidos solúveis totais dos frutos foram afetados significativamente nas diferentes condições de armazenamento, sendo a sua manutenção melhor na condição de armazenamento refrigerado com absorção de etileno (Ta- bela 1). A absorção de etileno do interior da câmara de armazenamento mantém a produção de etileno e a respiração dos frutos mais baixa, tendo como provável conseqüência a diminuição da utilização dos açúcares no processo de respiração e maturação. Por outro lado foi reportado que em mirtilos os teores de sólidos solúveis totais foram pouco influenciados pelo tempo (Jackson et al., 1999) e pelas condições de armazenamento (Harb \& Streif, 2004). Day et al. (1990) observaram que o conteúdo de SST em mirtilos manteve-se inalterado durante 12 semanas de armazenamento a $4^{\circ} \mathrm{C}$, devido à interação dinâmica entre as reações de anabolismo e catabolismo de carboidratos nos tecidos.

Maior porcentagem de frutos firmes, após sete semanas de armazenamento seguidas de três dias e meio a $20^{\circ} \mathrm{C}$, foi obtida com o armazenamento em atmosfera refrigerada (Tabela 1). Harb \& Streif (2006) também encontraram o melhor resultado quando os frutos de mirtilo ‘Bluecrop' foram armazenados em atmosfera refrigerada. Nessa condição observou-se no período de exposição a $20^{\circ} \mathrm{C}$ menor taxa respiratória e produção de etileno (Figura 1A e B). A diminuição desses processos resulta em diminuição da ação das pectinases, responsáveis pelo amaciamento e pela quebra das cadeias de ácidos poligalacturônicos, que dão firmeza e rigidez à parede celular da fruta. Segundo Fan et al. (1993), a firmeza da polpa de mirtilo 'Bluecrop' diminui com o aumento da pressão parcial de $\mathrm{CO}_{2}$ acima de $15 \mathrm{kPa}$. Schotsmans et al. (2007) observaram que durante o período de armazenamento de quatro semanas de mirtilos 'Centurion' armazenados em atmosfera refrigerada apresentaram maior firmeza, enquanto frutos do mesmo cultivar armazenados em atmosfera controlada $\left(2,5 \mathrm{kPa} \mathrm{O}_{2}\right.$ $+15 \mathrm{kPa} \mathrm{CO}_{2}$ ) apresentaram perda da firmeza. Estes autores também verificaram que em todo período de armazenamento a firmeza dos frutos armazenados em atmosfera refrigerada foi superior à dos frutos armazenados em atmosfera controlada. Forney et al. (2003)

Tabela 1. Características físicas e químicas de mirtilo ‘Bluegem’ mantido por 84 horas a $20^{\circ} \mathrm{C}$, após sete semanas em diferentes condições de armazenamento a $0,5^{\circ} \mathrm{C}$

\begin{tabular}{lcccc}
\hline $\begin{array}{c}\mathbf{O}_{2}+\mathbf{C O}_{2} \\
(\mathbf{k P a})\end{array}$ & $\begin{array}{c}\text { ATT } \\
\left(\mathbf{m e q ~ 1 0 0 ~} \mathbf{~ m L}^{-1}\right)\end{array}$ & $\begin{array}{c}\text { SST } \\
\text { ( }{ }^{\circ} \text { Brix) }\end{array}$ & $\begin{array}{c}\text { Firmeza } \\
\mathbf{( \% )}\end{array}$ & $\begin{array}{c}\text { Frutos murchos } \\
\text { (\%) }\end{array}$ \\
\hline $20,8+0,03(\mathrm{AR})$ & $8,14 \mathrm{ab}^{*}$ & $12,08 \mathrm{~b}$ & $59,52 \mathrm{a}$ & $9,66 \mathrm{a}$ \\
$20,8+0,03+\mathrm{AE}$ & $7,70 \mathrm{bcd}$ & $12,43 \mathrm{a}$ & $52,76 \mathrm{a}$ & $10,90 \mathrm{a}$ \\
$2,0+10,0$ & $7,51 \mathrm{~cd}$ & $12,03 \mathrm{~b}$ & $41,42 \mathrm{~b}$ & $8,41 \mathrm{ab}$ \\
$2,0+15,0$ & $7,75 \mathrm{bcd}$ & $11,88 \mathrm{~b}$ & $35,50 \mathrm{~b}$ & $5,63 \mathrm{c}$ \\
$10,0+15,0$ & $7,34 \mathrm{~d}$ & $11,40 \mathrm{c}$ & $37,17 \mathrm{~b}$ & $6,53 \mathrm{bc}$ \\
$2,0+10,0+1-\mathrm{MCP}$ & $8,17 \mathrm{a}$ & $11,45 \mathrm{c}$ & $39,70 \mathrm{~b}$ & $8,98 \mathrm{ab}$ \\
$\mathrm{CV} \%$ & 2,17 & 1,16 & 5,88 & 7,11 \\
\hline
\end{tabular}

Os frutos apresentaram, no momento da colheita, teores de ATT de 10,48meq $100 \mathrm{~mL}^{-1}$ e SST de $11,7^{\circ} \mathrm{Brix}$.

AE: absorção de etileno e 1-MCP: aplicação de $900 \mathrm{mg} \mathrm{kg}^{-1}$ de 1 -metilciclopropeno.

*Médias seguidas de mesma letra na vertical não diferem entre si pelo teste de Tukey $(p<0,05)$. 
reportaram que quando os frutos foram armazenados em pressões parciais de $\mathrm{CO}_{2}$ acima de $10 \mathrm{kPa}$ na temperatura de $0^{\circ} \mathrm{C}$, a firmeza diminuiu, enquanto que quando armazenados com zero ou $10 \mathrm{kPa}$ de $\mathrm{CO}_{2}$ a firmeza, após nove semanas de armazenamento, foi maior que a do início do armazenamento.

A menor ocorrência de frutos murchos foi obtida nas pressões parciais de $15 \mathrm{kPa} \mathrm{CO}_{2}$ (Tabela 1 ). Forney et al. (1998), trabalhando com o cultivar Burlington, também obtiveram menor quantidade de frutos murchos quando esses foram armazenados por nove semanas sob pressões parciais de $\mathrm{CO}_{2}$ entre 10 e $15 \mathrm{kPa}$. Estes autores verificaram que quando os frutos foram armazenados em atmosfera refrigerada durante nove semanas a quantidade de frutos murchos chegou a 31\%. Hardenburg et al. (1986) salientaram que frutos pequenos, como é o caso do mirtilo, tendem a apresentar alta taxa de desidratação durante o armazenamento refrigerado, devido à sua grande área de exposição.

A absorção de etileno promoveu aumentos nos teores de polifenóis totais e de antocianinas dos frutos (Tabela 2). Quando os frutos foram armazenados em baixas pressões parciais de $\mathrm{O}_{2}(2,0 \mathrm{kPa})$, o conteúdo total de polifenóis e antocianinas, em geral, foi inferior ao dos frutos submetidos ao armazenamento com altas pres- sões parciais de $\mathrm{O}_{2}$ (Tabela 2). Zheng et al. (2003) verificaram que atmosferas de armazenamento com pressões parciais de $\mathrm{O}_{2}$ maior ou igual a $60 \mathrm{kPa}$ proporcionaram aumento na concentração dos polifenóis totais e antocianinas, aumentando a atividade antioxidante. Estes autores também observaram que quanto maior o aumento da pressão parcial de $\mathrm{O}_{2}$, de $20,0 \mathrm{kPa}$ para $100 \mathrm{kPa}$, maiores foram os conteúdos dos polifenóis totais e de antocianinas. Connor et al. (2002) também observaram aumento do conteúdo de polifenóis totais, antocianinas e atividade antioxidante após três semanas de armazenamento em AR.

Dentre as condições avaliadas, o armazenamento em atmosfera refrigerada os frutos mantiveram melhor as qualidades física e químicas no período de pós-armazenagem, mantendo mais elevadas a acidez e SST, bem como a porcentagem de frutos firmes. Em tais condições apresentaram menor taxa respiratória e produção de etileno e ainda maior produção de polifenóis totais e antocianinas. A maior porcentagem de frutos murchos na condição de AR não diminuiu a qualidade dos frutos para o consumo. Não foi observado em nenhum tratamento incidência de podridões. Também não foi verificada diferença estatística para a perda de massa de frutos nos diferentes tratamentos (Tabela 2).

Tabela 2. Polifenóis totais, antocianinas e perda de massa durante o armazenamento de mirtilo 'Bluegem' mantido por 84 horas a $20^{\circ} \mathrm{C}$, após sete semanas em diferentes condições de armazenamento a $0,5^{\circ} \mathrm{C}$

\begin{tabular}{|c|c|c|c|}
\hline $\begin{array}{c}\mathrm{O}_{2}+\mathrm{CO}_{2} \\
(\mathbf{k P a})\end{array}$ & $\begin{array}{c}\text { Polifenóis totais } \\
\text { (mg de ác.gálico } L^{-1} \text { ) }\end{array}$ & $\begin{array}{c}\text { Antocianinas } \\
\left.\text { (mg de malvidina } L^{-1}\right)\end{array}$ & $\begin{array}{c}\text { Perda de massa } \\
(\%)\end{array}$ \\
\hline $20,8+0,03(\mathrm{AR})$ & 4937,8 bc* & $2456,4 \mathrm{ab}$ & $2,36 \mathrm{a}$ \\
\hline $20,8+0,03+\mathrm{AE}$ & 5515,3 a & 2644,8 a & $3,08 \mathrm{a}$ \\
\hline $2,0+10,0$ & $4745,3 \mathrm{c}$ & 2118,8 c & $2,51 \mathrm{a}$ \\
\hline $2,0+15,0$ & 4784,2 c & 2092,8 c & $2,91 \mathrm{a}$ \\
\hline $10,0+15,0$ & $5230,3 \mathrm{ab}$ & 2270,3 bc & $3,69 \mathrm{a}$ \\
\hline $2,0+10,0+1-\mathrm{MCP}$ & $5064,8 \mathrm{abc}$ & 2304,5 bc & $2,68 \mathrm{a}$ \\
\hline $\mathrm{CV} \%$ & 4,90 & 5,38 & 23,40 \\
\hline
\end{tabular}

Os frutos apresentaram no momento da colheita teores de polifenóis totais de 2.895,7mg de ác.gálico $\mathrm{L}^{-1}$ e antocianinas de $942,2 \mathrm{mg}$ de malvidina $\mathrm{L}^{-1}$. AE: subtração de etileno e 1-MCP: aplicação de $900 \mathrm{mg} \mathrm{kg}^{-1} \mathrm{de} 1$-metilciclopropeno.

*Médias seguidas de mesma letra na vertical não diferem entre si pelo teste de Tukey $(\mathrm{p}<0,05)$.

\section{CONCLUSÕES}

A melhor condição de conservação de mirtilo cv. Bluegem é o armazenamento refrigerado, e a absorção de etileno na câmara de armazenagem poderá trazer benefícios na manutenção da qualidade pós-colheita.

A atmosfera controlada com altas concentrações de $\mathrm{CO}_{2}$ reduz a qualidade pós-colheita dos frutos de mirtilo.

A utilização de 1-MCP não é recomendada, pela ineficiência na conservação da qualidade dos frutos após o armazenamento.

\section{REFERÊNCIAS}

Brackman A, Weber A, Pinto JAV, Neuwald DA \& Steffens CA (2008) Manutenção da qualidade pós-colheita de maçãs 'Royal Gala’ e ‘Galaxy’ sob armazenamento em atmosfera controlada. Ciência Rural, 38:2478-2484.

Connor AM, Luby JJ, Hancock JF, Berkheimer S \& Hanson EJ (2002) Changes in fruit antioxidant activity among blueberry cultivars during cold-temperature storage. Journal of Agriculture Food Chemistry, 50:893-898.

Day NB, Skura BJ \& Powrie WD (1990) Modified atmosphere packaging of blueberries: microbiological changes. Canadian Institute of Food Science and Technology Journal, 23:59-65. 
Di Stefano R, Cravero MC \& Gentilini N (1989) Metodi per lo studio dei polifenoli dei vini. L’Enotecnico, 25:83-89.

Fan X, Patterson ME, Robbins JA, Fellman JK \& Cavalieri RP (1993) Controlled atmosphere storage of 'Bluecrop' blueberries (Vaccinium corymbosum L.). In: $6^{\text {th }}$ International Controlled Atmosphere Research Conference, Ithaca, New York. Proceedings, Cornell University/Ithaca, v.2, p.699-704.

Forney CF, Jordan MA \& Nicholas KUKG (2003) Effect of CO on physical, chemical, and quality changes in 'Burlington' blueberries. Acta Horticulturae, 600:587-893.

Forney CF, Nicholas KUKG \& Jordan MA (1998) Effects of postharvest storage conditions on firmness of 'Burlington' blueberry fruit. 8th North American Research and Extension Workers Conference, Wilmington, North Carolina. Proceedings, Wilmington/NC, p.227-232.

Harb JY \& Streif J (2004) Controlled atmosphere storage of highbush blueberries cv. 'Duke'. European Journal of Horticultural Science, 69:66-72.

HarbJY \& Streif J (2006) Einfluss verschiedener Lagerbedingungen auf Haltbarkeit und Fruchtqualität von Heidelbeeren der Sorte 'Bluecrop’. Erwerbs-Obstbau, 48:115-120.

Hardenburg RE, Watada AE \& Wang CY (1986) The commercial storage of fruits, vegetables, and florist, and nursery stocks. Agriculture Handbook n.66, Washington, Unites States Department of Agriculture. 130p.

Jackson ED, Sanford KA, Lawrence RA, Mcrae KB \& Stark R (1999) Lowbush blueberry quality changes in response to prepacking delays and holding temperatures. Postharvest Biology and Technology, 15:117-126.

Milholland RD (1995) Anthracnose fruit rot (ripe rot). In: Caruso F \& Ramsdell D (Eds.) Compendium of blueberry and cranberry diseases. St. Paul Minn, American Phytopathological Society Press. 87p.

Miller WR, Mcdonald RE \& Crocker TE (1988) Fruit quality of rabbiteye blueberries as influenced by weekly harvests, cultivars, and storage duration. HortScience, 23:182-184.
Prange RK, Asiedu SK, DeEll JR \& Westgarth AR (1995) Quality of Fundy and Blomidon lowbush blueberries: Effects of storage atmosphere, duration and fungal inoculation. Canadian Journal of Plant Science, 75:479-483.

Schotsmans W, Molan A \& Mackay B (2007) Controlled atmosphere storage of rabbiteye blueberries enhances postharvest quality aspects. Postharvest Biology and Technology, 44:277-285.

Singleton VL \& Rossi JA (1965) Colorimetry of total phenolics with phosphomolybdic-phosphotungstic acid reagents. American Journal of Enology and Viticulture, 16:144-158.

Sisler EC \& Serek M (1999) Compounds controlling the ethylene receptor. Botanical Bulletin of Academia Sinica, 40:1-7.

Su MS \& Chien PJ (2007) Antioxidant activity, anthocyanins, and phenolics of rabbiteye blueberry (Vaccinium ashei) fluid products as affected by fermentation. Food Chemistry, 104:182-187.

Su MS \& Silva JL (2006) Antioxidant activity, anthocyanins, and phenolicsof rabbiteye blueberry (Vaccinium ashei) by-products as affected by fermentation. Food Chemistry, 97:447-451.

Warren JM, Walter WE \& Mainland CM (1973) Effects of ethephon upon fruit development and ripening of highbush blueberries in the greenhouse. Hortscience, 8:504-507.

Watkins CB (2000) Responses of horticultural commodities to high carbon dioxide as related to modified atmosphere packaging. HortTechnology, 10:501-506.

Westwood MN (1982) Fruticultura de zonas templadas. Barcelona, Mundi-Prensa, 461p.

Wills RBH \& Ku VVV (2002) Use of 1-MCP to extend the time to ripen of green tomatoes and postharvest life of ripe tomatoes. Postharvest Biology and Technology, 26:85-90.

Zheng Y, Wang CY, Wang SY \& Zheng W (2003) Effect of highoxygen atmospheres on blueberry phenolics, anthocyanins, and antioxidant capacity. Journal of Agricultural and Food Chemistry, 51:7162-7169. 